\title{
A 17-years old patient presented with clear cell carcinoma of uterine cervix
}

Brnčić-Fischer Alemka, Haller Heman, Perović Danko, Klarić Marko, Vejnović Danilo, Glavan-Gačanin Lana

\section{Clinical Hospital Centre Rijeka Department of Gynecology\&Obstetrics Rijeka, Croatia}

\section{Introduction}

A 17-year-old girl presented with the complaints of irregular menstrual bleeding for last five months. There was no maternal history of any drug/DES exposure during pregnancy and she had negative familial cancer history.

Transrectal ultrasound discovered a cervical tumor measuring $60 \times 50 \mathrm{~mm}$. Adnexal mass were presented on both ovaries, high vasculated. We didn't perform vaginal examination per speculam beacuse she had no sexual intercourse. Magnetic resonance imaging showed solid mass in the cervix with cystic components, solid mass of both ovaries and suspected pelvic lymph nodes. Biopsy confirmed the diagnosis of clear cell adenocarcinoma of cervix. Pap smear taken at biopsy showed adenocarcinoma and HPV testing was negative. CA 125 was elevated (74.5). Radical abdominal hysterectomy, bilateral salpingo-oophorectomy, systemic pelvic and paraaortic lymphadenectomy , infracolic omentectomy, appendectomy,resection of distal part of left urether with reimplantation (psoas hitch) was perfomed. Pathological examination showed pelvic and paraaortic lymph node involment, lymph-vascular space involment, penetration of cervical tumor in vagina and metastases in both ovaries, peritoneum and omentum. There was no residual tumor. Postoperative adjuvant chemotherapy (6 cycle of paclitaxel and carboplatin, in three- weeks intervals) and radiotherapy was administrated. Ten months after operation $\mathrm{Ca} 125$ level is negative (8.6) and there is no sign of malignant activity. After two months there has been a rise in tumor marker (CA 125) and PET CT confirmed the relaps of disease in liver, peritoenm, thoracal spine and lungs.

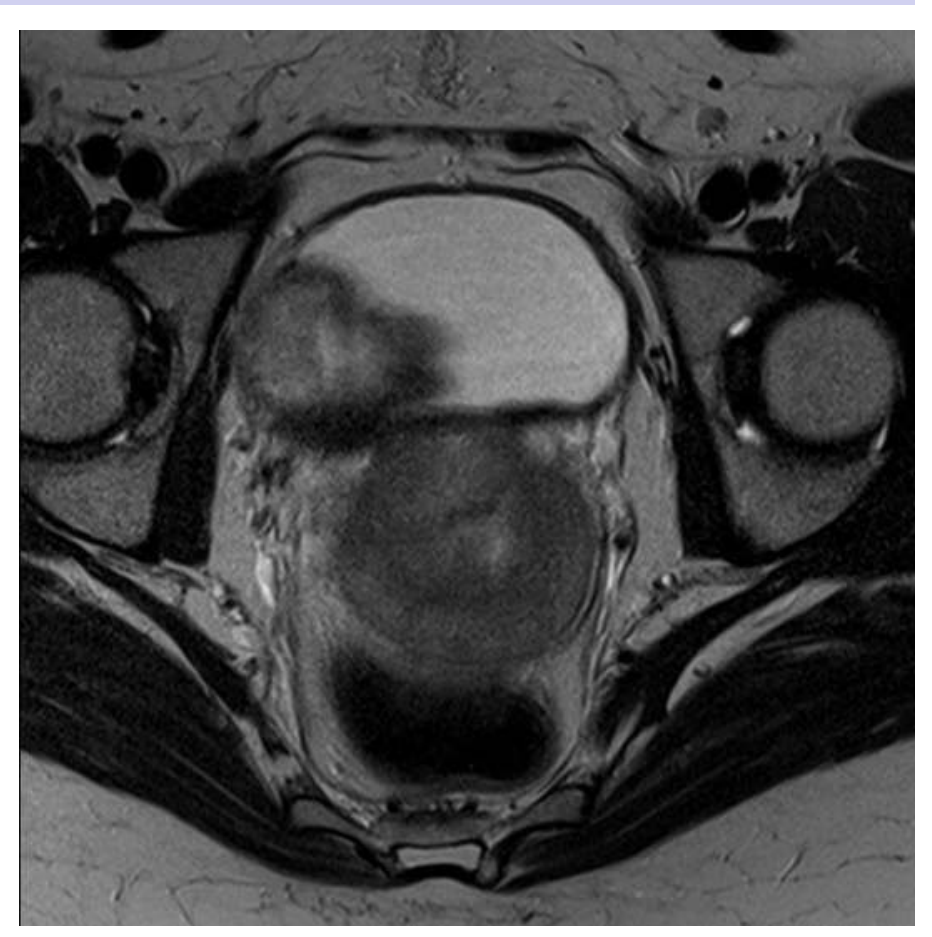

MRI of pelvis shows the mas on the cervix and ovary

\section{Discussion}

Primary clear cell carcinoma of cervix is extremely rare in women without in utero DES exposure and in such cases it concerns mostly postmenopausal women. Clear cell adenocarcinoma (CCAC) has a bimodal age distribution. The first peak occurs in women who are 17 to 37 years of age, and the second peak occurs in women who are 44 to 88 years of age. Liebrich et al. (Eur J Gynecol Oncol, 2009) have reported 18 cases of primary cervical cancer, persistently negative for high-risk HPV-DNA, in virgins and very young women. Only few studies have explored the association between HR HPV and CCAC. In these small studies HR HPV positivity varied between $0 \%$ and 100 (Pirog et a. AM J Pathol 2000; Waggoner et al, Obstet Gynecol 1994). CCAC in young virginal patients is often misdiagnosed as precocious puberty or anovulatory bleeding. Thomas reported that only six of 31 CCAC patients (18\%) had abnormal Pap tests (Gynecol Oncol 2008). Although CCAC is an uncommon tumor, it must be considered in the differential diagnosis in young women and children who have cervicovaginal lesions even without in utero DES exposure history. In these group of patients CCAC usually presents at advanced stage and diagnosis may be delayed beacuse of nonspecificity of symptoms, refusal of gynecological examination. No guidelines have been established for the treatment of CCAs, as their treatment is mainly based on experience with squamous cell carcinomas and adenocarcinomas.

\section{Conclusion}

CCAC in adolescents usually is often misdiagnosed because of its rarity. It should be considerd as a possible differential diagnosis in a case of vaginal bleeding, even without a history of sexual intercourse

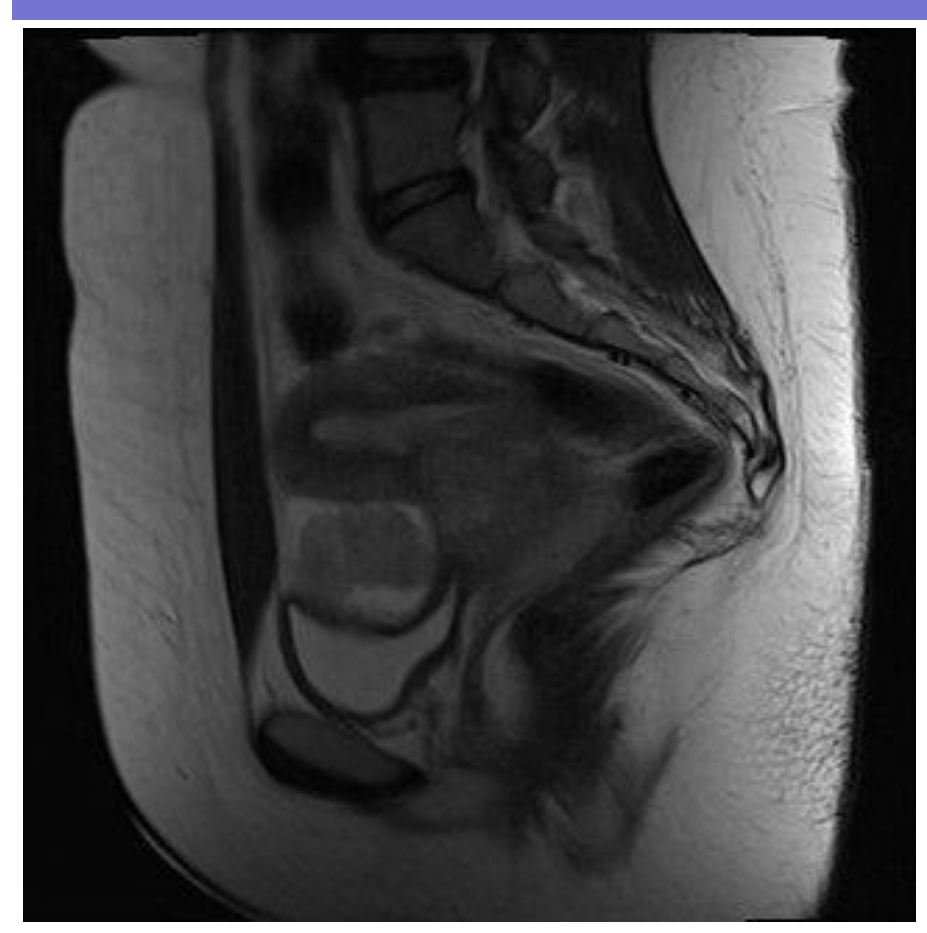

MRI of pelvis showing well defined mass involving the cervix 